\title{
INITIAL SERUM CREATININE AND NADIR CREATININE - PROGNOSTIC MARKERS FOR RENAL OUTCOME IN PATIENTS WITH PUV
}

\author{
Teodora Luchița ${ }^{1}$, Nicolae Sebastian Ionescu ${ }^{1,2}$
}

\begin{abstract}
Objective: This study aims to analyze the correlation between the initial creatinine and the nadir creatinine values and the risk of chronic kidney disease in patients with posterior urethral valves. Materials and methods: The medical records of all patients with PUV treated in the surgery department of The Emergency Children's Hospital "Marie Curie" between January 2007 - January 2020 were analyzed. The age at presentation, serum creatinine, nadir creatinine, and glomerular filtration rate were noted. The patients were divided in three groups based on the marker values. Results: 77 patients were studied. The high-risk group $(13 \%)$ was represented by patients with initial creatinine and nadir creatinine $>0.85 \mathrm{mg} / \mathrm{dL}$, with $100 \%$ progression to CKD. The low-risk group (33\%) showed a very low development of renal insufficiency for values of $<0.4 \mathrm{mg} / \mathrm{dL}$.
\end{abstract}

Keywords: posterior urethral valves, initial serum creatinine, nadir creatinine, chronic kidney disease

\section{Introduction}

Posterior urethral valves are the most common cause of lower urinary tract obstruction in children, with a broad spectrum of consequences on the bladder and kidneys. Despite the early treatment realized by valve ablation or urinary deviation, $25-40 \%$ of the patients develop chronic kidney disease $[1,2]$.

The study aims to analyze some prognostic factors associated with the worsening of renal function. The initial serum creatinine and the nadir creatinine are cheap and easy to obtain in any medical center. Knowing the risk of the patient to develop CKD can be very helpful in planning proper management for the patient and early parental counseling.

\section{Material and methods}

The study included registered and followed-up patients from the Emergency Children's Hospital "Marie Curie" Bucharest, between January 2007 and January 2019. The prognostic factors that were studied were: abnormal antenatal ultrasound, oligohydramnios, age at initial urinary drainage, initial serum creatinine value, nadir creatinine, and current renal function. The nadir creatinine value is defined as the lowest serum creatinine in the first 12 months after bladder drainage [1,3-5]. The value was recorded in $\mathrm{mg} / \mathrm{dL}$. The chronic kidney disease stage was determined by calculating the eGFR using the Schwartz formula. Chronic renal insufficiency was defined by stage- 2 or higher CKD according to the Kidney Disease Outcomes Quality Initiative guidelines (eGFR $<90 \mathrm{ml} / \mathrm{min}$ ) [6]. SPSS Statistics for Windows version 17.0 was used for statistical analysis. In order to establish the correlation between the creatinine value and the renal kidney disease, the Mann-Whitney U test and the Shapiro-Wilk test were used.

\section{Results and Discussions}

A number of 81 patients were identified, with available progress data in 77 patients. The mean age at diagnosis was 28.61 months (10 days - 156 months), IQR $(1,39)$. The median follow-up was 7.5 years. The mean age at the CKD was 52.01 months, IQR 17.5 (10.75-56.25).

Recorded initial creatinine ranged between 0.18 and $2.2 \mathrm{mg} / \mathrm{dL}$, while the nadir creatinine ranged between 0.2 and $1.2 \mathrm{mg} / \mathrm{dL}$. The patients were divided into three groups, depending on the initial creatinine and nadir creatinine values. In the first group (creatinine $<0.4 \mathrm{mg} / \mathrm{dL}$ ), no CKD during follow-up was seen. On the other hand, in the group of patients with initial creatinine higher than $0.85 \%, 8$ out of 10 patients developed CKD during the follow-up. In the middle group (creatinine between 0.45 and $0.85 \mathrm{mg} / \mathrm{dL}$ ) the ratio of patients with and without CKD was almost equal (Table 1). However, as the values of initial creatinine are lower and closer to $0.4 \mathrm{mg} / \mathrm{dL}$, the change of CKD is lower (Figure 1 and Figure 2).

The analysis of the nadir creatinine showed that only 3 out of 40 patients $(7.5 \%)$ developed chronic kidney disease during follow-up (Figure 3 and Figure 4). Therefore, a value lower than $0.4 \mathrm{mg} / \mathrm{dL}$ might be considered a prognostic factor for a good renal outcome. In the high-risk group, with nadir creatinine values $>0.85 \mathrm{mg} / \mathrm{dL}$, all the patients developed CKD. The data is available in table 2.

1"Carol Davila" University of Medicine and Pharmacy, Bucharest

2"M.S. Curie" Clinical Emergency Hospital for Children, Bucharest. "Carol Davila" University of Medicine and Pharmacy, Bucharest

E-mail: teodora.luchita@gmail.com,nsionescu@yahoo.com 


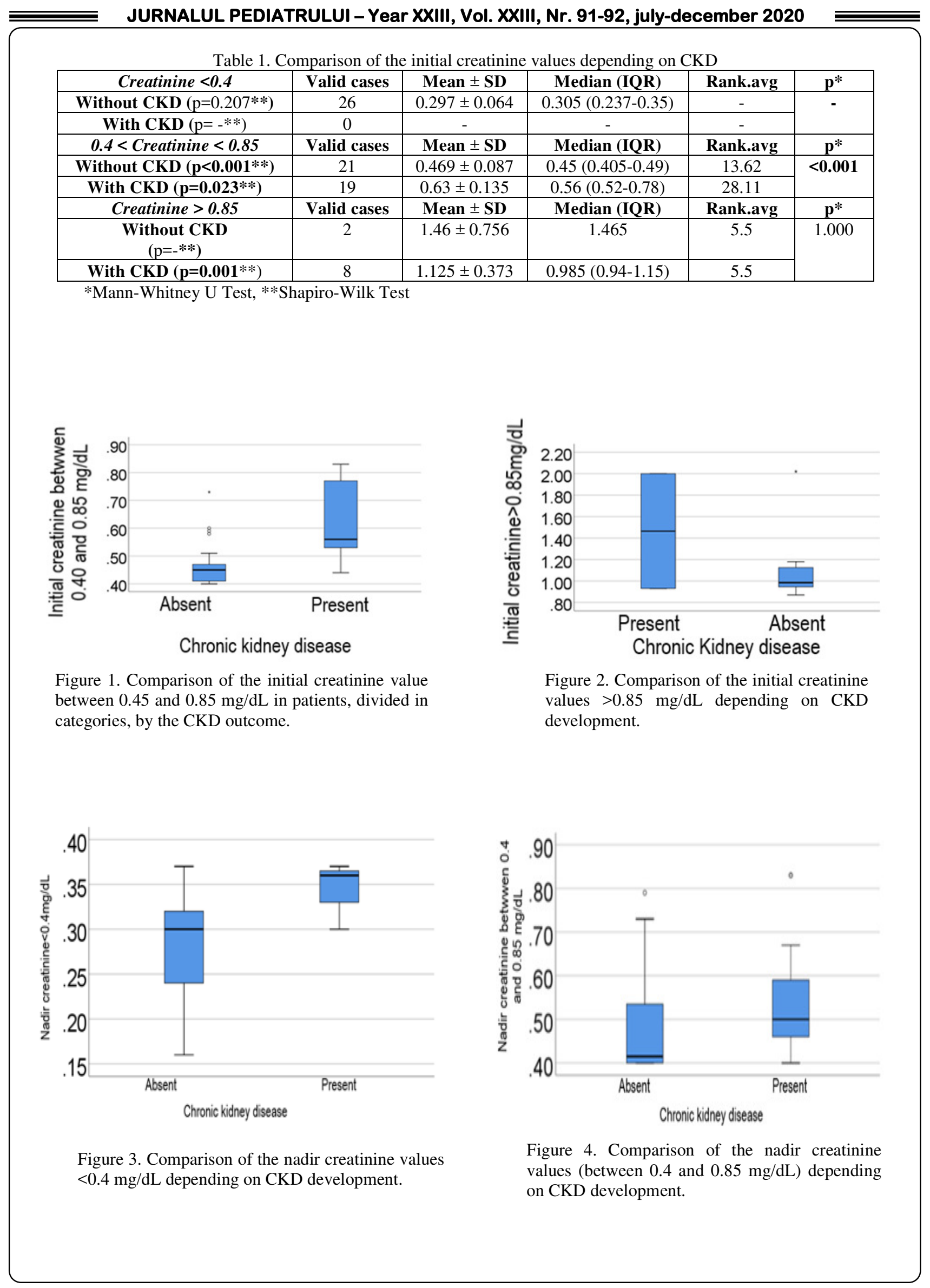


Table 2. Comparison of the nadir creatinine values depending on CKD.

\begin{tabular}{|c|c|c|c|c|c|}
\hline Nadir creatinine $<0.4$ & $\begin{array}{l}\text { Valid } \\
\text { cases }\end{array}$ & Mean \pm SD & Median (IQR) & Rank.avg & $\mathbf{p}^{*}$ \\
\hline Without CKD $(p=0.008 * *)$ & 37 & $\begin{array}{c}0.284 \pm \\
0.056\end{array}$ & $\begin{array}{c}0.3(0.235- \\
0.32)\end{array}$ & 19.53 & \multirow[t]{2}{*}{0.064} \\
\hline With CKD $\left(\mathrm{p}=0.253^{* *}\right)$ & 3 & $\begin{array}{c}0.343 \pm \\
0.037\end{array}$ & 0.36 & 32.5 & \\
\hline $\begin{array}{l}\text { Nadir creatinine between } 0.4 \text { and } 0.85 \\
\text { mg/dL }\end{array}$ & $\begin{array}{l}\text { Valid } \\
\text { cases }\end{array}$ & Mean \pm SD & Median (IQR) & Rank.avg & $\mathbf{p}^{*}$ \\
\hline Without CKD $(p=0.001 * *)$ & 12 & $\begin{array}{c}0.491 \pm \\
0.136\end{array}$ & $\begin{array}{l}0.415(0.4- \\
0.552)\end{array}$ & 12.21 & \multirow[t]{2}{*}{0.140} \\
\hline With CKD $(p=0.011 * *)$ & 17 & $\begin{array}{l}0.54 \pm \\
0.131\end{array}$ & $\begin{array}{l}0.5(0.455- \\
0.595)\end{array}$ & 16.97 & \\
\hline Nadir creatinine $>0.85 \mathrm{mg} / \mathrm{dL}$ & $\begin{array}{l}\text { Valid } \\
\text { cases }\end{array}$ & Mean \pm SD & Median (IQR) & Rank.avg & $\mathbf{p}^{*}$ \\
\hline Without CKD $(\mathrm{p}=-* *)$ & 0 & - & - & - & \multirow[t]{2}{*}{-} \\
\hline With CKD $\left(\mathrm{p}=0.263^{* *}\right)$ & 7 & $\begin{array}{c}1.021 \pm \\
0.093\end{array}$ & $1(0.97-1.08)$ & - & \\
\hline
\end{tabular}

*Mann-Whitney U Test, **Shapiro-Wilk Test

\section{Conclusions}

Initial serum creatinine and nadir creatinine are cheap and easy to obtain in any hospital. These markers were widely studied in literature as reliable markers for renal outcome in patients with PUV. Initial creatinine $<0.4 \mathrm{mg} / \mathrm{dL}$ was associated with no progression to renal insufficiency. The nadir creatinine value $<0.4 \mathrm{mg} / \mathrm{dL}$ was associated with CKD only in $7,5 \%$ of patients. Both markers higher than $0.85 \%$ were correlated with $100 \%$ progression to renal insufficiency.
They can guide the clinicians in establishing proper follow-up protocols and early parental guidance. However, other factors can lead to a poor renal outcome. Multiple urinary infections, untreated bladder-valve syndrome, coexistence of ureteral dilatation (due to vesicoureteral reflux or to ureterovesical junction stenosis) are factors that can contribute individually to chronic kidney disease development.

Conflict of interests: no conflict

\section{References}

1. W. DeFoor, C. Clark, E. Jackson, P. Reddy, E. Minevich, C. Sheldon, "Risk factors for end stage renal disease in children with posterior urethral valves". J Urol 2008;180(4 Suppl.):1705e8. Discussion 1708.

2. P. Yohannes, M. Hannah, "Current trends in the management of posterior urethral valves in the pediatric population". Urology 2002;60(6):947-53.

3. M.S. Ansari, A. Gulia, A. Srivastava, R. Kapoor, "Risk factors for progression to end-stage renal disease in children with posterior urethral valves". J Pediatr Urol 2010;6(3):261-4.

4. R. Coleman, T. King, C.D. Nicoara, M. Bader, L. McCarthy, H. Chandran, "Nadir creatinine in posterior urethral valves: How high is low enough? Journal of pediatric urology. 2015;11(6):356.1-5.

5. R. Coleman, T. King, C.D. Nicoara, M. Bader, L. McCarthy, H. Chandran, "Combined creatinine velocity and nadir creatinine: A reliable predictor of renal outcome in neonatally diagnosed posterior urethral valves". Journal of pediatric urology. 2015;11(4):214.13.

6. H.F. Parkhouse, C.R. Woodhouse, "Long-term status of patients with posterior urethral valves". Urol Clin North Am 1990;17(2):373-8.

\section{Correspondence to:}

Teodora Luchița

"Carol Davila" University of Medicine and Pharmacy,

Dionisie Lupu Street, no. 37, Sector 2, Bucharest

Phone: 0730039947

E-mail: teodora.luchita@gmail.com 